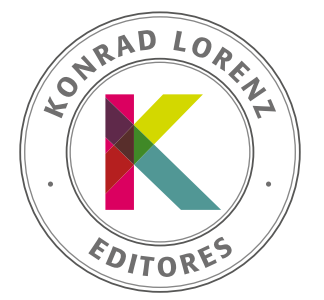

\title{
Propiedades psicométricas de la Escala de Ciber-Violencia en Parejas Adolescentes (Cib-VPA)
}

\author{
María-Jesús Cava ${ }^{\mathrm{a}, *}$ y Sofía Buelga ${ }^{\mathrm{a}}$
}

${ }^{a}$ Departamento de Psicología Social. Universidad de Valencia. Valencia, España.

Recibido el 6 de octubre de 2017; aceptado el 29 de noviembre de 2017

\author{
PALABRAS CLAVE \\ ciberviolencia \\ de pareja, \\ cibervictimización, \\ adolescentes, \\ escala, evaluación
}

\begin{abstract}
Resumen La utilización que los adolescentes hacen de las redes sociales, móviles e internet, para ejercer violencia hacia la pareja resulta preocupante. Por esta razón, es necesario disponer de instrumentos fiables, específicos para población adolescente y que permitan evaluar tanto situaciones de acoso como de victimización. El objetivo de este estudio fue analizar las propiedades psicométricas de la Escala de Ciber-violencia en Parejas Adolescentes (Cib-VPA). Esta escala consta de dos subescalas, ciberacoso perpetrado y cibervictimización, con 10 ítems cada una relativos a conductas agresivas y de control que pueden ser perpetradas o sufridas a través de redes sociales en parejas adolescentes. De una muestra inicial de 762 adolescentes, entre 12 y 18 años, aquellos que tenían pareja o la habían tenido en el último año cumplimentaron la escala Cib-VPA. La muestra final fue de 363 adolescentes (56.6\% chicas). Mediante análisis factorial se constataron dos factores similares en cada subescala: cibercontrol (conductas de excesivo control) y ciberagresión (amenazas e insultos a través de redes sociales). La fiabilidad de estos factores es adecuada en las dos subescalas. Se observan, además, correlaciones positivas entre cibercontrol y ciberagresión, perpetrados y sufridos, y conductas de violencia y victimización en parejas adolescentes fuera de las redes sociales.

(c) 2018 Fundación Universitaria Konrad Lorenz. Este es un artículo Open Access bajo la licencia CC BY-NC-ND (http://creativecommons.org/licenses/bync-nd/4.0/).
\end{abstract}

\section{Psychometric properties of the Cyber-Violence Scale in Adolescent Couples (Cib-VPA)}

Abstract The use that adolescents make of social networks, mobile and the Internet to perpetrate violence towards their couple is worrying. Hence, it is necessary to have reliable instruments specific to the adolescent population to facilitate the evaluation of both harassment and victimization situations. The objective of this study was to analyze the psychometric properties of the Scale of Cyber-violence in Adolescent Couples (Cib-VPA). This scale consists of two

* Autor para correspondencia.

Correo electrónico: Maria.J.Cava@uv.es 
sub-scales, Cyber-harassment perpetrated and Cyber-victimization, with 10 items related to aggressive and control behaviors that can be perpetrated or suffered through social networks in adolescent couples. From an initial sample of 762 adolescents aged 12-18, those who had a partner or had had a partner in the last year completed the Cib-VPA. The final sample included 363 adolescents (56.6\% girls). Factorial analysis confirmed two similar factors in each sub-scale: cyber-control (excessive control behaviors) and cyber-aggression (threats and insults through social networks). The reliability of these factors is adequate in the two subscales. Positive correlations between cyber-control and - perpetrated and suffered - cyber-aggression, violence and victimization behaviors in adolescent couples outside social networks are also observed.

(C) 2018 Fundación Universitaria Konrad Lorenz. This is an open access article under the CC BY-NCND license (http://creativecommons.org/licenses/bync-nd/4.0/).

En los últimos años se ha experimentado un gran cambio en la comunicación entre adolescentes, y el teléfono móvil e Internet son elementos centrales en sus relaciones interpersonales (Baker \& Carreño, 2016; Borrajo \& Gamez-Guadix, 2016; Ortega-Barón, Buelga, \& Cava, 2016). Las nuevas tecnologías de la información y la comunicación suponen importantes beneficios a nivel personal, social y económico; pero también han propiciado el desarrollo de problemas relacionados con el abuso y la agresión, como es el caso del cyberbullying (Buelga, Martínez, \& Cava, 2017; Zych, Ortega-Ruiz, \& Marín-López, 2016). En las parejas adolescentes las nuevas tecnologías se han integrado como parte fundamental de la relación, y estas incluyen los conflictos y las posibles agresiones (Borrajo, Gamez-Guadix, Pereda, \& Calvete, 2015; Schnurr, Mahatmya, \& Basche, 2013; Zweig, Dank, Yahner, \& Lachman, 2013), si bien el uso que los adolescentes hacen de las nuevas tecnologías para ejercer violencia contra la pareja constituye una temática muy poco investigada hasta el momento.

En relación con la violencia en parejas adolescentes fuera de las redes sociales, los estudios han mostrado una prevalencia muy elevada, sobre todo de la violencia verbal-emocional o psicológica, y una alta frecuencia de agresiones mutuas (Cava, Buelga, \& Carrascosa, 2015; Fernández-Fuertes \& Fuertes, 2010; Foshee \& Reyes, 2011; Leen et al., 2013). Para los adolescentes las primeras relaciones de pareja conllevan nuevos retos y nuevas formas de relacionarse, para las cuales carecen de experiencia previa (Ortega \& Sánchez, 2011; Viejo, 2014). En este contexto algunos adolescentes, influidos por ciertos mitos sobre el amor romántico, llegan a considerar los celos y el control a la pareja como una expresión de amor, lo que les dificulta identificar ciertas conductas tales como insultos, chantajes o amenazas como formas de violencia (Carrascosa, Cava, \& Buelga, 2016; Díaz-Aguado \& Carvajal, 2011). La violencia de pareja en adolescentes tiene graves consecuencias y se asocia con autoconcepto negativo, baja satisfacción con la vida, pobre rendimiento académico, elevado estrés, ansiedad, sintomatología depresiva, quejas somáticas, abuso de sustancias, desórdenes alimenticios y conductas sexuales de riesgo (Leen et al., 2013; Penado \& Rodicio-García, 2017; Romito, Beltramini, \& Escribà-Agüir, 2013; Shorey et al., 2012, 2015).

Aunque en las últimas décadas se han incrementado las investigaciones sobre la violencia en parejas adolescentes, las investigaciones sobre la violencia de pareja online, o ciberviolencia de pareja, en adolescentes son muy escasas (Peskin et al., 2017). Este tipo de violencia se produce entre adolescentes que son pareja o expareja mediante la utiliza- ción de medios tecnológicos (Peskin et al., 2017; Zweig et al., 2013). La ciberviolencia de pareja en adolescentes se ha relacionado con la violencia de pareja física y emocional (Temple et al., 2016; Yahner, Dank, Zweig, \& Lachman, 2015; Zweig et al., 2013) y ha sido considerada en algunas ocasiones como un subtipo de la violencia emocional (Korchmaros, Ybarra, Langhinrichsen-Rohling, Boyd, \& Lenhart, 2013). Sin embargo, la violencia online tiene algunas características particulares que la diferencian de la violencia cara a cara, como la ausencia de fronteras geográficas y temporales, la facilidad de acceso a la víctima y el elevado número de personas que pueden ver fotos y comentarios denigrantes de la víctima debido a la rápida difusión de la información que se produce en las redes sociales (Bennett, Guran, Ramos, \& Margolin, 2011; Buelga et al., 2017).

La ciberviolencia de pareja tiene, por tanto, características únicas que la diferencian de la violencia de pareja offline (fuera de las redes sociales) y que resaltan la necesidad de una investigación específica (Peskin et al., 2017). En este sentido, la ciberviolencia en parejas adolescentes puede ser extremadamente humillante, realizarse rápida y fácilmente, producirse de forma pública y ocurrir en cualquier lugar y en cualquier momento, incluso después de haber finalizado la relación de pareja (Bennett et al., 2011; Stonard, Bowen, Walker, \& Price, 2017; Zweig, Lachman, Yahner, \& Dank, 2014). Además, la experiencia de cibervictimización de pareja puede ser cualitativamente diferente para la víctima debido a características específicas, tales como la posible repetición continuada de la agresión al ser los mensajes digitales permanentes y poder compartirse (Korchmaros et al., 2013; Stonard et al., 2017; Zweig et al. 2014).

La ciberviolencia en parejas adolescentes, también denominada abuso online en el noviazgo (Borrajo \& Gámez-Guadix, 2016), incluye comportamientos realizados para controlar a la pareja o expareja a través de medios electrónicos, con conductas tales como visitar frecuentemente su perfil en redes sociales o controlar constantemente dónde está, con quién y qué hace, así como también conductas realizadas con la intención de causarle daño mediante mensajes insultantes, amenazantes o difundiendo información negativa sobre la pareja o expareja (Borrajo et al., 2015; Borrajo \& Gámez-Guadix, 2016, Darvell, Walsh, \& White, 2011; Van Ouytsel, Ponnet, \& Walrave, en prensa).

Una de las dificultades para avanzar en la investigación sobre la ciberviolencia en parejas adolescentes es la falta de instrumentos adecuados para su medición. En este sentido, es necesario disponer de instrumentos que estén adaptados a parejas adolescentes y que, además, considerando 
la alta prevalencia de agresiones mutuas en estas parejas (Fernández-Fuertes \& Fuertes, 2010; Foshee \& Reyes, 2011; Leen et al., 2013), permitan evaluar tanto la violencia ejercida como sufrida. Sin embargo, los instrumentos disponibles son escasos y algunos se centran solo en la perpetración de la violencia o en la victimización (Bennet et al., 2011; Fox \& Warber, 2013), sin permitir una evaluación completa de esta problemática. Entre los instrumentos que evalúan perpetración y victimización de violencia de pareja online se encuentra el Controlling Partners Inventory (CPI, Burke, Wallen, Vail-Smith, \& Knox, 2011), que consta de cuatro factores: Control a la pareja mediante cámara, fotos, GPS y spyware, Comunicación excesiva, Amenazas y Conductas controladoras. También evalúan perpetración y victimización la escala de Ciber dating abuse de Zweig et al. (2013) con dos subescalas - Ciberacoso sexual y Ciberacoso no sexual-; y el Ciber Dating Violence Inventory (CDVI, Morelli, Bianchi, Chirumbolo, \& Baiocco, 2017), que incluye dos factores, Violencia psicológica, que integra conductas como insultar a la pareja o amenazarla con dejar la relación, y Violencia relacional, relativo a conductas dirigidas a dañar las relaciones sociales de la pareja.

En lengua española son muy escasos los instrumentos existentes. En este sentido, la escala Cyberdating Q_A desarrollada por Sánchez, Muñoz-Fernández y Ortega-Ruíz (2015) evalúa la calidad de las relaciones de pareja en adolescentes a través de las redes sociales, midiendo aspectos positivos tales como la intimidad entre la pareja y aspectos negativos como los celos. Si bien esta escala aporta una información muy útil sobre las relaciones románticas adolescentes y la influencia de las redes sociales en ellas, no permite una evaluación conjunta de la violencia perpetrada y sufrida en la relación de pareja. Por otra parte, la escala de Abuso online en el noviazgo de Borrajo et al. (2015) sí incluye una evaluación de la violencia online perpetrada y sufrida, pero está centrada en parejas entre 18 y 30 años, y convendría disponer de un instrumento específico para parejas más jóvenes.

Teniendo en cuenta esta necesidad, se desarrolló la escala de Ciber-violencia en parejas adolescentes (Cib-VPA). Esta escala incluye ítems relativos a conductas de control excesivo y de agresión directa a través de las redes sociales, tanto perpetrada como sufrida, en parejas adolescentes. El objetivo del presente estudio fue analizar las propiedades psicométricas de esta escala, explorando su estructura factorial, su fiabilidad y su validez de constructo mediante el análisis de sus relaciones con la violencia de pareja perpetrada y sufrida por los adolescentes fuera de las redes sociales.

\section{Método}

\section{Participantes}

La muestra inicial de esta investigación estuvo compuesta por 762 adolescentes (50.7\% chicas, $49.3 \%$ chicos), entre 12 y 18 años $(M=14.06$; $D T=1.81)$. Estos adolescentes eran estudiantes de Enseñanza Secundaria en dos institutos públicos de Valencia (España). Esta muestra formaba parte de un estudio más amplio sobre ajuste psicosocial en adolescentes. A partir de esta muestra inicial, únicamente aquellos adolescentes que tenían pareja en ese momento, o que la habían tenido en el último año, cumplimentaron las escalas relativas a violencia de pareja a través de redes sociales y violencia de pareja fuera de las redes sociales. Para cumplimentar estas escalas se indicó a los adolescentes que pensaran en su última relación de pareja, caso de haber tenido varias en el último año. Se indicó a los adolescentes que debían considerar como una relación de pareja aquellas relaciones que fueran percibidas por ellos como una relación romántica y que, por tanto, supusieran o hubieran supuesto para ellos una implicación afectiva.

La muestra final de este estudio estuvo formada por 363 adolescentes (56.6\% chicas, $43.3 \%$ chicos), entre 12 y 18 años $(M=14.34 ; D T=1.73)$. El $96.4 \%$ de los adolescentes informaron de relaciones de pareja heterosexuales y un $3.6 \%$ de relaciones homosexuales. La edad media de sus parejas fue 15.02 años $(D T=2.80)$. En cuanto a la duración de la relación, la mayoría de los adolescentes (un 53.5\%) informaron de relaciones entre 1 y 6 meses de duración, siendo menores los porcentajes de parejas con una duración menor a un mes (17.4\%), entre 6 meses y 1 año (15.7\%) y superiores a 1 año (13.4\%). El porcentaje de adolescentes cuya relación de pareja es menor a un mes se corresponde, sobre todo, con adolescentes menores de 14 años. Estos datos son coincidentes con la mayor brevedad que caracteriza a las relaciones de pareja durante la adolescencia temprana, unas primeras relaciones románticas que, aunque habitualmente breves, son de gran importancia para los adolescentes (Connolly, Craig, Goldberg, \& Pepler, 2004). Así, si bien en estudios sobre violencia en parejas de mayor edad, suelen desestimarse las relaciones de pareja con una duración menor a un mes, en los estudios que incluyen la adolescencia temprana es conveniente considerar también las relaciones de menor duración.

\section{Instrumentos}

La Escala de Ciber-violencia en Parejas Adolescentes (CibVPA) fue desarrollada para evaluar tanto situaciones de violencia perpetrada como de violencia sufrida a través de las redes sociales en parejas adolescentes, utilizando para ello dos subescalas diferentes. Una primera subescala, denominada Ciberacoso perpetrado, incluye ítems relativos a conductas agresivas y de control perpetradas contra la pareja a través de las redes sociales (e.g., "He insultado o amenazado por privado a mi chico/a", "Estoy pendiente de si mi chico/a está en línea en el móvil o conectado en redes sociales"). La segunda subescala, denominada Cibervictimización, describe estas mismas conductas agresivas y de control, pero en este caso evalúa en qué medida los adolescentes han sufrido dichas conductas en su relación de pareja (e.g., "Mi chico/a me ha insultado o amenazado por privado", "Mi chico/a está pendiente de si estoy en línea en el móvil o conectado en redes sociales"). A estos ítems, los adolescentes responden mediante una escala Likert de cuatro puntos ( 1 = nunca; 2 = algunas veces; 3 = bastantes veces; 4 = siempre).

Para la elaboración de esta escala se realizó previamente una revisión de otras escalas previas similares y se entrevistó a cuatro adolescentes que conocían casos de ciberviolencia de pareja. A partir de estas entrevistas y contando con la colaboración de cinco expertos en adolescencia y ciberacoso, se elaboró una escala inicial compuesta por un 
total de 44 ítems paralelos, 22 ítems referidos a conductas agresivas y de control perpetradas contra la pareja y 22 ítems que incluían estas mismas conductas, pero referidos a victimización. El contenido de los ítems fue revisado por estos expertos, siguiendo criterio interjueces para su redacción definitiva. En esta revisión del contenido por parte de los expertos se siguieron pautas de confidencialidad entre jueces, desconocimiento de la información elaborada por los otros jueces, envío y recepción de la información a través de medios diferidos y tiempo limitado de resolución de 15 días para cada juez. Posteriormente, se llevó a cabo una prueba piloto con 20 adolescentes que cumplimentaron la escala completa e informaron de dificultades en la comprensión de algunos ítems. Teniendo en cuenta los resultados de esta prueba piloto, se eliminaron los ítems que presentaban mayores dificultades. En la elaboración final de la escala se tuvo en cuenta, además, que los ítems fueran paralelos en cuanto a su contenido en ambas subescalas. La escala Cib-VPA quedó conformada, finalmente, por dos subescalas (Ciberacoso perpetrado y Cibervictimización), con 10 ítems cada una.

Por otra parte, la violencia de pareja perpetrada y sufrida por los adolescentes fuera de redes sociales se evaluó mediante el CADRI (Conflict in Adolescent Dating Relationships Inventory de Wolfe et al., 2001; adaptación española: Fernández-Fuertes, Fuertes, \& Pulido, 2006). Se han utilizado en este estudio seis subescalas: Violencia física, compuesta por cuatro ítems (e.g., "He empujado o zarandeado a mi pareja") que evalúan comportamientos violentos de tipo físico, como golpes o empujones; Violencia verbal-emocional o psicológica, integrada por 10 ítems ("He insultado a mi pareja con frases de desprecio") relativos a amenazas, insultos o chantajes; Violencia Relacional, compuesta por tres ítems (e.g., "Saqué a relucir algo malo que él/ella había hecho en el pasado") que evalúan el daño ejercido a través de conductas como la difusión de rumores en el entorno social; Victimización física, cuatro ítems (e.g., "Mi pareja me empujó o me zarandeó”) que evalúan comportamientos violentos de tipo físico sufridos por parte de la pareja; Victimización verbal-emocional o psicológica, 10 ítems (e.g., "Mi chico/a me insultó con frases de desprecio") relativos a amenazas, insultos o chantajes sufridos; y Victimización Relacional, tres ítems (ejemplo: “Mi pareja sacó a relucir algo malo que yo había hecho en el pasado") que evalúan conductas realizadas con el fin de dañar las relaciones sociales de la víctima. Los ítems tienen cuatro opciones de respuesta: nunca, rara vez (1 o 2 ocasiones), a veces (entre 3 y 5 veces) y con frecuencia (6 o más ocasiones). En este estudio su fiabilidad fue .67 Violencia física, .84 Violencia verbal-emocional, .59 Violencia relacional, .87 Victimización física, .90 Victimización verbal-emocional y .75 Victimización relacional.

\section{Procedimiento}

En primer lugar, se seleccionaron dos centros educativos de la ciudad de Valencia (España), ubicados en zonas de nivel socioeconómico medio. Una vez seleccionados, se envió a estos centros una carta informativa, explicándoles los objetivos del proyecto y solicitando su colaboración. Posteriormente, se contactó telefónicamente con los directores de estos centros y se concertó con ellos una entrevista para explicarles más detenidamente el proyecto y resolver posi- bles dudas sobre la investigación. En la entrevista, además de explicar el proyecto, se estableció el compromiso con estos centros de, una vez concluida la investigación, informarles sobre los principales resultados obtenidos y asesorarles sobre prevención de la violencia en adolescentes. En esta reunión se les entregaron también los consentimientos informados para que fueran cumplimentados por los padres de los adolescentes, junto con una carta explicativa para las familias. En esta carta se les indicaba que el objetivo de la investigación era analizar las relaciones de los adolescentes con sus iguales, tanto relaciones de amistad como primeras relaciones de pareja, y conocer posibles dificultades derivadas tanto del aislamiento social como de situaciones de violencia, con el fin último de prevenir las dificultades y favorecer el desarrollo positivo de los adolescentes. Asimismo, se les indicó que toda la información relativa a sus hijos sería confidencial, que sus respuestas serían anónimas y que podían consultar cualquier duda con el equipo investigador. Únicamente, un $2 \%$ de las familias no autorizaron que sus hijos participaran en esta investigación.

Tras la obtención de todos los permisos correspondientes, se realizó la aplicación de los instrumentos. Esta aplicación fue realizada por investigadores previamente entrenados, en las aulas habituales de los alumnos. Los adolescentes fueron informados de que su participación en la investigación era voluntaria y anónima, y que podían abandonar el estudio en cualquier momento. Se les agradeció su participación y su sinceridad en las respuestas, y se les indicó que, una vez cumplimentados, si deseaban más información sobre el proyecto o necesitaban ayuda específica en relación con la violencia de pareja, podían contactar con los investigadores. En el desarrollo de este estudio se siguieron las normas y valores éticos demandados en la investigación con seres humanos por la APA, respetando asimismo los principios fundamentales de la Declaración Helsinki, en sus actualizaciones y normativas vigentes. Esta investigación fue aprobada por el Comité de Ética de la Universidad de Valencia (H1456762885511).

\section{Análisis de datos}

Se llevó a cabo, en primer lugar, un análisis factorial exploratorio con cada una de las dos subescalas (Ciberacoso perpetrado y Cibervictimización) por separado, utilizando el paquete estadístico SPSS (versión 24). En ambas subescalas se exploró, previamente, por separado la idoneidad de realizar este análisis mediante la prueba de esfericidad de Barlett y la medida de adecuación de la muestra de Kaiser-Meyer-Olkin (KMO). Una vez observado que en ambas subescalas era adecuada la realización del análisis factorial exploratorio, se realizó este análisis en cada sub-escala por separado. En ambos análisis factoriales exploratorios, para la extracción de los factores se utilizó el método de Ejes Principales, con rotación Oblimin. Se utilizó la rotación Oblimin, puesto que se consideraba probable que existieran correlaciones significativas entre los factores.

Posteriormente, y puesto que existía una correlación importante entre los factores obtenidos mediante análisis factorial exploratorio en ambas subescalas, se contrastó mediante análisis factorial confirmatorio tanto un posible modelo bifactorial como también un posible modelo unifactorial. Estos análisis factoriales confirmatorios fueron realizados 
por separado con cada subescala (Ciberacoso perpetrado y Cibervictimización), utilizando el programa MPlus (Muthén \& Muthén, 1998-2010). Debido a la desviación de la multinormalidad de los datos, se utilizaron estimadores robustos (WLSMV) para determinar la bondad de ajuste del modelo. El método de Mínimos Cuadrados Ponderados Robustos (WLSMV) es, además, el método más adecuado con variables ordinales que presentan cierto incumplimiento del supuesto de la normalidad. Por otra parte, y teniendo en cuenta que se desaconseja utilizar una única medida de ajuste global del modelo (Bentler, 2007), se calcularon varios índices: $\chi^{2}$, Root Mean Square Error of Approximation (RMSEA), Comparative Fit Index (CFI) y Tucker-Lewis Index (TLI). Un modelo se considera que ajusta bien a los datos observados cuando la ratio entre el estadístico $\chi^{2}$ y los grados de libertad es menor a tres, los índices de ajuste son iguales o superiores a .95 y el RMSEA es menor a .08 .

Una vez analizada la estructura factorial de ambas subescalas, se calculó la fiabilidad de los factores y se realizaron análisis de correlación bivariada (Pearson) para explorar las relaciones entre estos factores y las variables de violencia de pareja (física, verbal-emocional y relacional) perpetrada y sufrida en parejas adolescentes fuera de las redes sociales.

\section{Resultados}

\section{Análisis Factorial Exploratorio}

Los resultados de la prueba de Kaiser-Meyer-Olkin $(\mathrm{KMO}=$ .864 para la sub-escala de Ciberacoso perpetrado y $\mathrm{KMO}=$ .913 para la subescala de Cibervictimización) y de la prueba de esfericidad de Barlett $\left(\chi^{2}=2225.74, g l=45, p<.001\right.$ para la subescala de Ciberacoso perpetrado; $\chi^{2}=2169.12$, $g l=45, p<.001$ para la subescala de Cibervictimización) fueron satisfactorios, por lo que se realizó el Análisis Factorial Exploratorio. Mediante este análisis se obtuvieron en la primera subescala (Ciberacoso perpetrado) dos factores que explican en su conjunto el $65.60 \%$ de la varianza (ver tabla 1). El primer factor, denominado ciberagresión, explica el $54.69 \%$ de varianza e incluye ítems relativos a amenazas, insultos y mensajes o fotos difundidos en las redes sociales para dañar a la víctima. El segundo factor, denominado cibercontrol, explica el $10.91 \%$ de varianza e incluye ítems relativos a conductas de control a la pareja perpetradas a través de las redes sociales, controlando por ejemplo si está conectada o no, qué fotos personales comparte o con qué amigos chatea. Estos dos factores (ciberagresión y cibercontrol) tienen una correlación de .541.

Por otra parte, el Análisis Factorial Exploratorio realizado con la segunda subescala (Cibervictimización) mostró también dos factores que explican en su conjunto el $70.55 \%$ de la varianza (tabla 1). El primer factor, ciberagresión, explica el $60.04 \%$ de varianza e incluye ítems relativos a amenazas e insultos de la pareja recibos online, y mensajes, comentarios o fotos difundidos por la pareja en redes sociales para dañarles. El segundo factor, denominado cibercontrol, explica el $10.50 \%$ de varianza e incluye ítems que hacen referencia a conductas de control online ejercidas por la pareja, tales como exigir que se conteste inmediatamente a llamadas y mensajes, supervisar si se está o no en línea y con quién se habla o se comparten comentarios o fotos en redes sociales. En esta subescala de Ciber-victimización, ambos factores (ciberagresión y cibercontrol) tienen una correlación de .641.

Por otra parte, respecto a las saturaciones de los ítems en cada factor, en la tabla 1 podemos observar cómo los ítems paralelos, es decir, aquellos que están referidos a un mismo tipo de conducta agresiva o de control (ya sea perpetrada en el caso de la subescala de Ciberacoso perpetrado o sufrida en el caso de la subescala de Ciber-victimización) presentan una estructura similar de saturación en los dos factores (ciberagresión y cibercontrol) obtenidos en ambas subescalas. Así, los cinco primeros ítems de ambas subescalas (Ciberacoso perpetrado y Cibervictimización) muestran saturaciones superiores a .40 únicamente en el factor denominado cibercontrol y los cinco últimos ítems de ambas subescalas presentan saturaciones superiores a .40 sólo en el factor denominado ciberagresión, siendo este criterio considerado adecuado para mantener un ítem en un factor (Worthington \& Whitttaker, 2006). No obstante, en el caso del ítem 4 de la subescala de Ciberacoso perpetrado su saturación en el factor de ciberagresión está muy cerca del valor .40. Por ello, en los posteriores análisis factoriales confirmatorios se contrastará tanto un modelo bifactorial incluyendo a este ítem, como también un modelo bifactorial eliminando este ítem.

\section{Análisis Factorial Confirmatorio}

Puesto que ambos factores (ciberagresión y cibercontrol) tienen correlaciones elevadas entre sí en las dos subescalas (Ciberacoso perpetrado y Cibervictimización) se contrastaron en ambas subescalas por separado tanto un modelo bifactorial como un modelo unifactorial. Además, en el caso de la subescala de Ciberacoso perpetrado se contrastó también un modelo bifactorial excluyendo el ítem 4. Los resultados de estos análisis se muestran en la tabla 2 . En esta tabla puede observarse que en ambas subescalas el modelo bifactorial ofrece un mejor ajuste a los datos, al aportar este modelo una ratio entre $\chi^{2}$ y los grados de libertad menor, valores mayores para CFI y TLI, y siendo en este modelo menor el valor de la RMSEA. Respecto al ítem 4 de la subescala de Ciberacoso perpetrado, los resultados obtenidos indican un mejor ajuste de los datos al modelo manteniendo este ítem. Por tanto, se asume para ambas subescalas un modelo bifactorial y se mantiene el ítem 4 en la subescala de Ciberacoso perpetrado.

A continuación, en la tabla 3 se muestran las cargas factoriales de los ítems de ambas subescalas en los factores de cibercontrol y ciberagresión, obtenidas en el análisis factorial confirmatorio contrastando el modelo bifactorial y manteniendo el ítem 4 en la subescala de Ciberacoso perpetrado. Tanto en la subescala de Ciberacoso perpetrado como en la subescala de Cibervictimización, puede observarse como los ítems relativos a control a la pareja y agresión directa se integran en sus respectivos factores con una carga factorial significativa.

\section{Fiabilidad y Análisis de Correlación}

A continuación, se analizó la fiabilidad de los dos factores obtenidos (cibercontrol y ciberagresión) en cada una de las dos subescalas (Ciberacoso perpetrado y Cibervictimización), 
Tabla 1 Análisis Factorial Exploratorio de las subescalas de Ciberacoso perpetrado y Cibervictimización (saturaciones de los ítems en cada factor)

\begin{tabular}{|c|c|c|}
\hline & $\begin{array}{l}\text { F1 } \\
\text { Ciberagresión }\end{array}$ & $\begin{array}{l}\text { F2 } \\
\text { Cibercontrol }\end{array}$ \\
\hline \multicolumn{3}{|l|}{ Subescala Ciberacoso perpetrado } \\
\hline 1. Me enfado si veo que mi chico/a está en línea y no me contesta enseguida & -.163 & .929 \\
\hline 2. Estoy pendiente de si mi chico/a está en línea en el móvil o conectado en redes sociales & .011 & .662 \\
\hline $\begin{array}{l}\text { 3. No dejo a mi chico/a chatear con algunos amigos/as, y si lo hace me enfado y le hago } \\
\text { sentir mal }\end{array}$ & .310 & .459 \\
\hline $\begin{array}{l}\text { 4. Le he hecho eliminar o bloquear amigos/as de sus redes sociales o de su móvil para que } \\
\text { no tenga contacto con ellos }\end{array}$ & .395 & .401 \\
\hline $\begin{array}{l}\text { 5. Me pongo celoso/a por comentarios, fotos o videos suyos en redes sociales y se los } \\
\text { hago eliminar }\end{array}$ & .216 & .556 \\
\hline 6. He contado rumores o mentiras sobre mi chico/a en redes sociales & .781 & .111 \\
\hline 7. He insultado o amenazado por privado a mi chico/a & .937 & -.005 \\
\hline $\begin{array}{l}\text { 8. Le he dicho a mi chico/a que si corta conmigo, diré o publicaré en redes sociales cosas } \\
\text { personales suyas }\end{array}$ & .943 & -.060 \\
\hline $\begin{array}{l}\text { 9. He hecho comentarios públicos sobre mi chico/a en Internet o en grupos del Whatsapp } \\
\text { que le han hecho sentir mal }\end{array}$ & .838 & -.015 \\
\hline $\begin{array}{l}\text { 10. He enviado o subido a redes sociales fotos, videos o mensajes suyos que no quería que } \\
\text { la gente viese sin su permiso }\end{array}$ & .926 & -.014 \\
\hline \multicolumn{3}{|l|}{ Subescala Cibervictimización } \\
\hline 1. Mi chico/a se enfada si ve que estoy en línea y no le contesto enseguida & -.084 & .858 \\
\hline 2. Mi chico/a está pendiente de si estoy en línea en el móvil o conectado en redes sociales & -.083 & .908 \\
\hline 3. No me deja chatear con algunos amigos/as y se enfada si lo hago & .113 & .788 \\
\hline $\begin{array}{l}\text { 4. Me ha hecho eliminar o bloquear amigos/as de mis redes sociales o de mi móvil para } \\
\text { que no tenga contacto con ellos }\end{array}$ & .314 & .475 \\
\hline $\begin{array}{l}\text { 5. Mi chico/a me ha hecho eliminar comentarios, fotos o videos míos en redes sociales } \\
\text { porque le ponían celoso/a }\end{array}$ & .271 & .614 \\
\hline 6. Ha contado rumores o mentiras sobre mí en redes sociales & .846 & -.021 \\
\hline 7. Mi chico/a me ha insultado o amenazado por privado & .814 & .109 \\
\hline $\begin{array}{l}\text { 8. Me ha dicho a que si corto con él/ella, dirá o publicará en redes sociales cosas } \\
\text { personales mías }\end{array}$ & .957 & -.060 \\
\hline $\begin{array}{l}\text { 9. Mi chico/a ha hecho comentarios públicos sobre mí en Internet o en grupos del } \\
\text { Whatsapp que me han hecho sentir mal }\end{array}$ & .780 & .035 \\
\hline $\begin{array}{l}\text { 10. Mi chico/a ha enviado o subido a redes sociales sin mi permiso fotos, videos o mensajes } \\
\text { míos que yo no quería que la gente viese }\end{array}$ & .870 & .012 \\
\hline
\end{tabular}

mediante el cálculo del coeficiente omega. Este coeficiente resulta más adecuado que otros, como el alpha de Cronbach, cuando se utilizan escalas ordinales. Los resultados de estos análisis mostraron adecuados índices de fiabilidad para todos los factores, obteniéndose en la subescala de Ciberacoso perpetrado una fiabilidad de .94 para el factor de cibercontrol y .97 para el factor de ciberagresión. En la subescala de Cibervictimización, el factor de cibercontrol mostró una fiabilidad de .92 y el factor de ciberagresión de .97.
Por último, se consideró la validez de constructo de la escala analizando las correlaciones entre los dos factores (cibercontrol y ciberagresión) que componen cada subescala (Ciberacoso perpetrado y Cibervictimización) y otros constructos con los que teóricamente se encuentran relacionados. En concreto, se analizaron las correlaciones entre estos factores y la violencia en parejas adolescentes, ya sea física, psicológica o relacional, perpetrada y sufrida fuera de redes sociales. Los resultados se muestran en la tabla 4. 
Tabla 2 Índices de bondad de ajuste de los modelos analizados

\begin{tabular}{|c|c|c|c|c|c|c|}
\hline Modelos & $\chi^{2}$ & $g l$ & $p$ & CFI & TLI & RMSEA \\
\hline \multicolumn{7}{|l|}{ Subescala Ciberacoso perpetrado } \\
\hline Modelo unifactorial (sin ítem 4) & 111.36 & 27 & $<.001$ & .967 & .956 & $\begin{array}{c}0.10 \\
(0.08-0.11)\end{array}$ \\
\hline Modelo unifactorial & 126.77 & 35 & $<.001$ & .969 & .960 & $\begin{array}{c}0.09 \\
(0.07-0.10)\end{array}$ \\
\hline Modelo bifactorial (sin ítem 4) & 75.61 & 26 & $<.001$ & .981 & .973 & $\begin{array}{c}0.07 \\
(0.06-0.09)\end{array}$ \\
\hline Modelo bifactorial & 64.53 & 34 & $<.001$ & .990 & .986 & $\begin{array}{c}0.05 \\
(0.03-0.07)\end{array}$ \\
\hline \multicolumn{7}{|l|}{ Subescala Cibervictimización } \\
\hline Modelo unifactorial & 121.93 & 35 & $<.001$ & .975 & .968 & $\begin{array}{c}0.09 \\
(0.07-0.10)\end{array}$ \\
\hline Modelo bifactorial & 87.37 & 34 & $<.001$ & .985 & .980 & $\begin{array}{c}0.07 \\
(0.05-0.08)\end{array}$ \\
\hline
\end{tabular}

En la tabla previa se observan correlaciones positivas significativas entre la violencia de pareja, tanto ejercida como sufrida por los adolescentes, fuera de redes sociales y la violencia de pareja a través de las redes sociales, ya sea mediante el control o la agresión. Todas las variables correlacionan además de forma muy elevada y se destaca, entre otras, la alta correlación obtenida entre la violencia física ejercida contra la pareja fuera de las redes sociales y la ciberagresión a la pareja $(r=.84 ; p<.001)$, entre la violencia física sufrida fuera de las redes y la ciberagresión a la pareja $(r=.84 ; p<.001)$, entre la violencia psicológica ejercida contra la pareja fuera de las redes y el cibercontrol a la pareja $(r=.83 ; p<.001)$ y entre la violencia de pareja psicológica sufrida por los adolescentes fuera de las redes sociales y el control sufrido a través de las redes $(r=.84 ; p<.001)$.

\section{Discusión}

El objetivo de este estudio fue analizar las propiedades psicométricas de la escala de Ciberviolencia en parejas adolescentes (Cib-VPA), con el fin de disponer de un instrumento fiable que permita evaluar la violencia perpetrada y sufrida en parejas adolescentes a través de las redes sociales. En este sentido, los resultados obtenidos avalan la utilidad y fiabilidad de este instrumento. En cuanto a su estructura factorial, los resultados del presente estudio confirman la existencia de dos factores, cibercontrol y ciberagresión, que englobarían las principales conductas de acoso a la pareja realizadas por los adolescentes a través de las redes sociales. Estos mismos dos factores, relativos al control y a las agresiones directas, han sido constatados en investigaciones previas sobre ciberviolencia en parejas jóvenes de más edad (Borrajo et al., 2015; Borrajo \& Gámez-Guadix, 2016).

Los dos factores obtenidos tienen, además, una alta coherencia desde el punto de vista teórico. Así, el factor de cibercontrol incluye conductas de excesivo control a la pa- reja realizadas a través de las redes sociales, con conductas tales como controlar constantemente si la pareja está o no conectada, con quién habla a través de las redes, a qué grupos pertenece, qué fotos y comentarios publica, e incluso obligando a la pareja a eliminar fotos publicadas o bloquear amistades. Estas conductas de excesivo control son las formas más frecuentes de ciberviolencia de pareja (Darvell et al., 2011; Reed, Tolman, \& Ward, 2017), y su utilización en adolescentes se ha relacionado con estereotipos de género rígidos y con la observación de conductas de control en otras parejas próximas (Van Ouytsel et al., en prensa). El factor de ciberagresión engloba conductas de agresión directa a la pareja a través de las redes sociales, con conductas tales como amenazarla, insultarla, difundir rumores o publicar comentarios ofensivos sobre ella. La prevalencia de las conductas de ciberagresión a la pareja es también elevada en población adolescente (Stonard, Bowen, Lawrence, \& Price, 2014), y puede tener graves consecuencias para las víctimas (Stonard et al., 2017; Zweig et al., 2014).

Además, cabe señalar que estos dos factores, cibercontrol y ciberagresión, han sido confirmados tanto en la subescala de violencia perpetrada online contra la pareja (Ciberacoso perpetrado) como en la subescala de victimización de pareja online (Cibervictimización). La escala Cib-VPA permite, por tanto, evaluar ambas formas de violencia de pareja online, el cibercontrol y la ciberagresión, tanto si son perpetradas como sufridas por los adolescentes. Ciertamente, es importante poder evaluar tanto la victimización como la perpetración de la ciberviolencia de pareja, puesto que en las parejas adolescentes las agresiones mutuas son frecuentes (Cava et al., 2015; Fernández-Fuertes \& Fuertes, 2010; Leen et al., 2013; Stonard et al., 2014), y esta característica podría trasladarse también a las redes sociales. Además, la conexión existente entre la violencia de pareja fuera y dentro de las redes sociales se ha visto confirmada en este estudio por las correlaciones constatadas entre ambas formas de violencia en adolescentes. 
Tabla 3 Análisis Factorial Confirmatorio de las subescalas de Ciberacoso perpetrado y Cibervictimización (modelo bifactorial)

\begin{tabular}{rc}
\hline F2 & F1 \\
Cibercagresión Cibercontrol
\end{tabular}

Subescala Ciberacoso perpetrado

1. Me enfado si veo que mi chico/a está en línea y no me contesta enseguida

2. Estoy pendiente de si mi chico/a está en línea en el móvil o conectado en redes sociales

3. No dejo a mi chico/a chatear con algunos amigos/as, y si lo hace me enfado y le hago sentir mal

4. Le he hecho eliminar o bloquear amigos/as de sus redes sociales o de su móvil para que no tenga contacto con ellos

5. Me pongo celoso/a por comentarios, fotos o videos suyos en redes sociales y se los hago eliminar

6. He contado rumores o mentiras sobre mi chico/a en redes sociales

0.866

7. He insultado o amenazado por privado a mi chico/a

0.991

8. Le he dicho a mi chico/a que si corta conmigo, diré o publicaré en redes sociales cosas personales suyas

9. He hecho comentarios públicos sobre mi chico/a en Internet o en grupos del Whatsapp que le han hecho sentir mal

10. He enviado o subido a redes sociales fotos, videos o mensajes suyos que no quería que la gente viese sin su permiso

\section{Subescala Cibervictimización}

1. Mi chico/a se enfada si ve que estoy en línea y no le contesto enseguida

2. Mi chico/a está pendiente de si estoy en línea en el móvil o conectado en redes sociales

3. No me deja chatear con algunos amigos/as y se enfada si lo hago

4. Me ha hecho eliminar o bloquear amigos/as de mis redes sociales o de mi móvil para que no tenga contacto con ellos

5. Mi chico/a me ha hecho eliminar comentarios, fotos o videos míos en redes sociales porque le ponían celoso/a

6. Ha contado rumores o mentiras sobre mí en redes sociales

0.918

7. Mi chico/a me ha insultado o amenazado por privado

8. Me ha dicho a que si corto con él/ella, dirá o publicará en redes sociales cosas personales mías

9. Mi chico/a ha hecho comentarios públicos sobre mí en Internet o en grupos del Whatsapp que me han hecho sentir mal

10. Mi chico/a ha enviado o subido a redes sociales sin mi permiso fotos, videos o mensajes míos que yo no quería que la gente viese

0.949

En estudios previos se han observado relaciones entre la violencia de pareja física y emocional fuera de las redes sociales y la ciberviolencia de pareja (Temple et al., 2016; Yahner et al., 2015; Zweig et al., 2013). En el presente estudio se han confirmado estas relaciones, y además se han constatado también relaciones entre la violencia de pareja de tipo relacional ejercida fuera de redes sociales y la ciberviolencia de pareja; así como entre las diversas formas de victimización de pareja fuera de las redes sociales y la cibervictimización. Estos resultados señalan la importante continuidad que los adolescentes mantienen entre el mun- do real y el mundo virtual, y cómo las conductas violentas fuera de las redes sociales se incorporan a las redes sociales. Esta misma continuidad entre espacio real y virtual ha sido constatada en el caso del bullying y el cyberbullying (Ortega-Barón, Buelga, Cava, \& Torralba, 2017). En futuras investigaciones deberá profundizarse en los vínculos que los adolescentes establecen entre la violencia dentro y fuera de redes sociales, así como en el papel desempeñado por ciertas variables personales y sociales que podrían contribuir conjuntamente a la violencia en ambos contextos. Así, por ejemplo, variables tales como las actitudes negativas 
Tabla 4 Correlaciones entre Ciberviolencia de pareja (Ciberacoso perpetrado y Cibervictimización) y Violencia de pareja (física, psicológica y relacional) perpetrada y sufrida fuera de redes sociales

\begin{tabular}{lcccc}
\hline & \multicolumn{2}{c}{ Ciberacoso perpetrado } & \multicolumn{2}{c}{ Cibervictimizacion } \\
& cibercontrol & ciberagresión & cibercontrol & ciberagresión \\
\hline Violencia de pareja física perpetrada & .80 & .84 & .76 & .81 \\
Violencia de pareja psicológica perpetrada & .83 & .73 & .78 & .69 \\
Violencia de pareja relacional perpetrada & .77 & .82 & .79 & .77 \\
Violencia de pareja física sufrida & .81 & .84 & .84 & .79 \\
Violencia de pareja psicológica sufrida & .82 & .75 & .78 \\
Violencia de pareja relacional sufrida & .77 & .78 & .81 \\
\hline
\end{tabular}

Nota: Todas las correlaciones son significativas

${ }^{*} p<.001$

hacia la autoridad y la normas, que han sido vinculadas en trabajos previos con la violencia que los adolescentes muestran fuera de redes sociales (Carrascosa, Cava, \& Buelga, 2015), podrían también estar relacionadas con su implicación en diversos tipos de violencia online.

El presente estudio tiene también algunas limitaciones. En este sentido, aunque el tamaño de la muestra utilizada en esta investigación es adecuado para los análisis realizados (Kline, 2015; Worthington \& Whittaker, 2006), sería conveniente llevar a cabo posteriores análisis de validación con muestras más amplias y que representen en mayor medida a los adolescentes españoles. También, aunque en este estudio se aporta evidencia sobre aspectos importantes relativos a las propiedades psicométricas del instrumento (validez factorial, validez convergente y fiabilidad), otros aspectos psicométricos como el análisis de la fiabilidad test-retest no han sido considerados y convendría que se analizaran en futuros estudios. Igualmente, sería conveniente analizar en futuras investigaciones la invarianza factorial de esta escala utilizando para ello muestras de adolescentes más amplias y de diferentes contextos sociales y culturales.

No obstante, y a pesar de estas limitaciones, el presente estudio avala la idoneidad de la escala Cib-VPA en investigaciones sobre ciberviolencia en parejas adolescentes. Dado el preocupante aumento que este tipo de violencia entre adolescentes ha experimentado en los últimos años, las investigaciones sobre sus factores explicativos y sus conexiones con otras formas de violencia online deberían incrementarse. Asimismo, en futuras investigaciones sería necesario continuar explorando las posibles diferencias de género en las conductas de ciberviolencia de pareja, así como analizar posibles diferencias en las motivaciones, percepciones y consecuencias que estas conductas tienen para chicos y chicas adolescentes. La escala Cib-VPA puede contribuir a incrementar las investigaciones en este ámbito, al ser un instrumento fiable y adecuado para evaluar la ciberviolencia de pareja, perpetrada y sufrida, en población adolescente. Las graves consecuencias que la violencia de pareja tiene para los adolescentes (Carrascosa et al., 2016; Reed et al., 2017; Stonard et al., 2017) avalan la necesidad de profundizar en su estudio, con el fin de desarrollar programas de prevención dirigidos específicamente a chicos y chicas en el inicio de sus primeras relaciones románticas. La intervención temprana con adolescentes puede, ade- más, evitar que este tipo de conductas se mantengan en futuras relaciones de pareja en edad adulta.

\section{Referencias}

Baker, C. K., \& Carreño, P. K. (2016). Understanding the role of technology in adolescent dating and dating violence. Journal of Child and Family Studies, 25, 308-320. http://dx.doi.org/10.1007/ s10826-015-0196-5

Bentler, P. M. (2007). On tests and indices for evaluating structural models. Personality and Individual Differences, 42(5), 825-829. http://dx.doi.org/10.1016/j.paid.2006.09.024

Bennett, D. C., Guran, E. L., Ramos, M. C., \& Margolin, G. (2011). College students' electronic victimization in friendships and dating relationships: Anticipated distress and associations with risky behaviors. Violence and Victims, 26, 410-429.

Borrajo, E., \& Gámez-Guadix, M. (2016). Abuso “online” en el noviazgo: Relación con depresión, ansiedad y ajuste diádico. Psicología Conductual, 24(2), 221-235.

Borrajo, E., Gámez-Guadix, M., Pereda, N., \& Calvete, E. (2015). The development and validation of the cyber dating abuse questionnaire among young couples. Computers in Human Behavior, 48, 358-365. http://dx.doi.org/10.1016/j.chb.2015.01.063

Buelga, S., Martínez, B., \& Cava, M.J. (2017). Differences in family climate and family communication among cyberbullies, cybervictims, and cyber bully-victims in adolescents. Computers in Human Behavior, 76, 164-173. http://dx.doi.org/10.1016/j. chb.2017.07.017

Burke, S. C., Wallen, M., Vail-Smith, K., \& Knox, D. (2011). Using technology to control intimate partners: An exploratory study of college undergraduates. Computers in Human Behavior, 27, 1162-1167. https://doi.org/10.1016/j.chb.2010.12.010

Carrascosa, L, Cava, M. J., \& Buelga, S. (2016). Ajuste psicosocial en adolescentes víctimas frecuentes y víctimas ocasionales de violencia de pareja. Terapia Psicológica, 34(2), 93-102.

Carrascosa, L., Cava, M. J., \& Buelga, S. (2015). Actitudes hacia la autoridad y violencia entre adolescentes: Diferencias en función del sexo. Suma Psicológica, 22, 102-109. http://dx.doi. org/10.1016/j.sumpsi.2015.08.003

Cava, M. J., Buelga, S., \& Carrascosa, L. (2015). Violencia física y psicológica ejercida en parejas adolescentes: Relación con el autoconcepto y la violencia entre iguales. Psicología Conductual, 23(3), 429-446.

Connolly, J. A., Craig, W., Goldberg, A., \& Pepler, D. (2004). Mixedgender groups, dating, and romantic relationships in early adolescence. Journal of Research on Adolescence, 14(2), 185207. http://dx.doi.org/10.1111/j.1532-7795.2004.01402003.x 
Darvell, M., Walsh, S., \& White, K. (2011). Facebook tells me so: Applying the theory of planned behavior to understand partner-monitoring behavior on Facebook. Cyberpsychology, Behavior, and Social Networking. 14(12), 717-722. https://doi. org/10.1089/cyber.2011.0035

Díaz-Aguado, M. J. \& Carvajal, I. (Dirs.) (2011). Igualdad y prevención de la violencia de género en la adolescencia y la juventud. Madrid, España: Ministerio de Sanidad, Igualdad y Servicios Sociales.

Draucker, C. B., \& Martsolf, D. S. (2010). The role of electronic communication technology in adolescent dating violence. Journal of Child and Adolescent Psychiatric Nursing, 23(3), 133-142. https://doi.org/10.1111/j.1744-6171.2010.00235.x

Fernández-Fuertes, A. A., \& Fuertes, A. (2010). Physical and psychological aggression in dating relationships of Spanish adolescents: Motives and consequences. Child Abuse \& Neglect, 34, 183-191. https://doi.org/10.1016/j.chiabu.2010.01.002

Fernández-Fuertes, A. A., Fuertes, A., \& Pulido, F. (2006). Evaluación de la violencia en las relaciones de pareja de los adolescentes. Validación del Conflict in Adolescent Dating Relationships Inventory (CADRI)-versión española. International Journal of Clinical and Health Psychology, 6, 339-358.

Foshee, V. \& Reyes, H. (2011). Dating abuse: Prevalence, consequences and causes. En J. R. Levesque (Eds.), Encyclopedia of Adolescence (pp. 602-615). New York, NY: Springer.

Fox, J. \& Warber, K.M. (2013). Romantic relationship development in the age of Facebook: An exploratory study of emerging adults' perceptions, motives, and behaviors. Cyberpsychology, Behavior, and Social Networking, 16(1), 3-7. https://doi.org/10.1089/ cyber.2012.0288

Kline, R.B. (2015). Principles and practice of structural equation modeling. New York, NY: Guilford.

Korchmaros, J.D., Ybarra, M. L., Langhinrichsen-Rohling, J., Boyd, D., \& Lenhart, A. (2013). Perpetration of teen dating violence in a networked society. Cyberpsychology, Behavior and Social Networking, 16, 561-567. https://doi.org/10.1089/cyber.2012.0627

Leen, E., Sorbring, E., Mawer, M., Holdsworth, E., Helsing, B., \& Bowen, E. (2013). Prevalence, dynamic risk factors and the efficacy of primary interventions for adolescent dating violence: An international review. Aggression and Violent Behavior, 18, 159-174. https://doi.org/10.1016/j.avb.2012.11.015

Morelli, M., Bianchi, D., Chirumbolo A., \& Baiocco, R. (2017). The cyber dating violence inventory. Validation of a new scale for online perpetration and victimization among dating partners. European Journal of Developmental Psychology, 1-8. http:// dx.doi.org/10.1080/17405629.2017.1305885

Muthén, B.O., \& Muthén, L.K. (1998-2010). Mplus user's guide (6th ed.). Los Angeles, CA: Muthén \& Muthén.

Ortega, R., \& Sánchez, V. (2011). Juvenile dating and violence. En C. P. Monks \& I. Coyne (Eds). Bullying in different contexts (pp. 113-136). Londres, Inglaterra: Cambridge University Press.

Ortega-Barón, J., Buelga, S., \& Cava, M. J. (2016). Influencia del clima escolar y familiar en adolescentes, víctimas de ciberacoso. Comunicar, 46, 57-65. http://dx.doi.org/10.3916/C46-2016-06

Ortega-Barón, J., Buelga, S., Cava, M. J., \& Torralba, E. (2017). Violencia escolar y actitud hacia la autoridad de estudiantes agresores de cyberbullying. Revista de Psicodidáctica, 22(1), 23-28. http://dx.doi.org/10.1387/RevPsicodidact.16398

Penado, M. \& Rodicio-García, M. L. (2017). Análisis del autoconcepto en las víctimas de violencia de género entre adolescentes. Suma Psicológica, 24, 107-114. http://dx.doi.org/10.1016/j. sumpsi.2017.08.001
Peskin, F. M., Markham, C. M., Shegog, R., Temple, J. R., Baumler, E. R., Addy, R. C. ... Emery, S. T. (2017). Prevalence and correlates of the perpetration of cyber dating abuse among early adolescents. Journal of Youth and Adolescence, 46, 358-375. http://dx.doi.org/10.1007/s10964-016-0568-1

Reed, L. A., Tolman, R. M., \& Ward, L. M. (2017). Gender matters: Experiences and consequences of digital dating abuse victimization in adolescent dating relationships. Journal of Adolescence, 59, 79-89. http://dx.doi.org/10.1016/j.adolescence.2017.05.015

Romito, P., Beltramini, L., \& Escribà-Agüir, V. (2013). Intimate partner violence and mental health among Italian adolescents: gender similarities and differences. Violence Against Women, 19(1), 89-106. http://dx.doi.org/10.1177/1077801212475339

Sánchez, V., Muñoz-Fernández, N., \& Ortega-Ruíz, R. (2015). “Cyberdating Q A": An instrument to assess the quality of adolescent dating relationships in social networks. Computers in Human Behavior, 48, 78-86. http://dx.doi.org/10.1016/j.chb.2015.01.006

Schnurr, M. P., Mahatmya, D., \& Basche, R. A. (2013). The role of dominance, cyber aggression perpetration, and gender on emerging adults' perpetration of intimate partner violence. Psychology of Violence, 3(1), 70-83. http://dx.doi.org/10.1037/ a0030601

Shorey, R. C., Seavey, A. E., Brasfield, H., Febres, J., Fite, P. J., \& Stuart, G. L. (2015). The moderating effect of social support from a dating partner on the association between dating violence victimization and adjustment. Violence Against Women, 21(4), 460-477. http://dx.doi.org/10.1177/1077801215570482

Shorey, R. C., Temple, J. R., Febres, J., Brasfield, H., Sherman, A. E., \& Stuart, G. L. (2012). The consequences of perpetrating psychological aggression in dating relationships: a descriptive investigation. Journal of Interpersonal Violence, 27(15), 29802998. http://dx.doi.org/10.1177/0886260512441079

Stonard, K. E., Bowen, E., Lawrence, T. R., \& Price, S. A. (2014). The relevance of technology to the nature, prevalence and impact of Adolescent Dating Violence and Abuse: A research synthesis. Aggression and Violent Behavior, 19, 390-417. http://dx.doi. org/10.1016/j.avb.2014.06.005

Stonard, K. E., Bowen, E., Walker, K., \& Price, S. A. (2017). "They'll always find a way to get to you": Technology use in adolescent romantic relationships and its role in dating violence and abuse. Journal of Interpersonal Violence, 32(14), 2038-2117. http://dx.doi.org/10.1177/0886260515590787

Temple, J. R., Choi, H. J., Brem, M., Wolford-Clevenger, C., Stuart, G. L., Peskin, M. F., \& Elmquist, J. (2016). The temporal association between traditional and cyber dating abuse among adolescents. Journal of Youth and Adolescence, 45, 340-349. http://dx.doi.org/10.1007/s10964-015-0380-3

Van Ouytsel, J. V., Ponnet, K., \& Walrave, M. (en prensa). Cyber dating abuse: Investigating digital monitoring behaviors among adolescents from a social learning perspective. Journal of Interpersonal Violence. http://dx.doi.org/10.1177/0886260517719538

Viejo, C. (2014). Violencia física en las relaciones sentimentales adolescentes: hacia la comprensión del fenómeno. Infancia y Aprendizaje, 37(4), 785-815. http://dx.doi.org/10.1080/021037 02.2014.977110

Wolfe, D. A., Scott, K., Reitzel-Jaffe, D., Wekerle, C., Grasley, C., \& Pittman, A. L. (2001). Development and validation of the conflict in adolescent dating relationships inventory. Psychological Assessment, 13, 277-293. http://dx.doi.org/10.1037/0022-006X. 71.2.279 
Worthington, R. L. \& Whittaker, T. A. (2006). Scale development research: a content analysis and recommendations for best practices. The Counselling Psychologist, 34(6), 806-838. http:// dx.doi.org/10.1177/0011000006288127

Yahner, J., Dank, M., Zweig, J. M., \& Lachman, P. (2015). The co-occurrence of physical and cyber dating violence and bullying among teens. Journal of Interpersonal Violence, 30(7), 10791089. http://dx.doi.org/10.1177/0886260514540324

Zweig, J. M., Dank, M., Yahner, J., \& Lachman, P. (2013). The rate of cyber dating abuse among teens and how it relates to other forms of teen dating violence. Journal of Youth and Adolescence, 42, 1063-1077. http://dx.doi.org/10.1007/s10964-013$9922-8$
Zweig, J. M., Lachman, P., Yahner, J., \& Dank, M. (2014). Correlates of cyber dating abuse among teens. Journal of Youth and Adolescence, 43, 1306-1321. http://dx.doi.org/10.1007/s10964013-0047-x

Zych, I., Ortega-Ruiz, R., \& Marín-López, I. (2016). Cyberbullying: a systematic review of research, its prevalence and assessment issues in Spanish studies. Psicología Educativa, 22(1), 5-18. http://dx.doi.org/10.1016/j.pse.2016.03.002 